\title{
Gata6 in pluripotent stem cells enhance the potential to differentiate into cardiomyocytes
}

\author{
Chang-Hwan Yoon ${ }^{1}$, Tae-Won Kim ${ }^{2}$, Seok-Jin $\mathrm{Koh}^{1}$, Young-Eun Choi ${ }^{3}$, Jin Hur ${ }^{3}$, Yoo-Wook Kwon ${ }^{3}$, Hyun-Jai Cho ${ }^{3}$ \& \\ Hyo-Soo Kim $^{2,3, *}$ \\ ${ }^{1}$ Cardiovascular Center \& Department of Internal Medicine, Seoul National University Bundang Hospital, Seongnam 13620, ${ }^{2}$ Molecular \\ Medicine \& Biopharmaceutical Sciences, Graduate School of Convergence Science and Technology, Seoul National University, Seoul 03080 , \\ ${ }^{3}$ Innovative Research Institute for Cell Therapy, Seoul National University Hospital, Seoul 03080, Korea
}

\begin{abstract}
Pluripotent stem cell (PSC) variations can cause significant differences in the efficiency of cardiac differentiation. This process is unpredictable, as there is not an adequate indicator at the undifferentiated stage of the PSCs. We compared global gene expression profiles of two PSCs showing significant differences in cardiac differentiation potential. We identified 12 up-regulated genes related to heart development, and we found that 4 genes interacted with multiple genes. Among these genes, Gata6 is the only gene that was significantly induced at the early stage of differentiation of PSCs to cardiomyocytes. Gata6 knock-down in PSCs decreased the efficiency of cardiomyocyte production. In addition, we analyzed $6 \mathrm{mESC}$ lines and 3 iPSC lines and confirmed that a positive correlation exists between Gata6 levels and efficiency of differentiation into cardiomyocytes. In conclusion, Gata6 could be utilized as a biomarker to select the best PSC lines to produce PSC-derived cardiomyocytes for therapeutic purposes. [BMB Reports 2018; 51(2): 85-91]
\end{abstract}

\section{INTRODUCTION}

Ischemic heart disease and heart failure are leading causes of death in many countries. Transplantation of stem cells, capable of regenerating the damaged heart, provides an attractive option for the acutely injured heart or end-stage heart failure (1). Among various stem cells, pluripotent stem cells (PSCs), including embryonic stem cells (ESCs) and induced pluripotent stem cells (iPSCs), are emerging as promising sources for cell therapy (2). Previous reports

${ }^{*}$ Corresponding author. Tel: +82-2-2072-2226; Fax: +82-2-7668904; E-mail: usahyosoo@gmail.com, hyosoo@snu.ac.kr

https://doi.org/10.5483/BMBRep.2018.51.2.176

Received 5 September 2017, Revised 13 October 2017, Accepted 30 October 2017

Keywords: Cardiomyocyte, Cell differentiation, GATA6 transcription factor, Stem cells demonstrated the feasibility to induce differentiation of ESC or iPSC toward cardiomyocytes and then to transplant them into an infarcted heart to repair the damage (3-5).

In the meantime, we also tried cardiac differentiation of those two PSCs (ESC and iPSC) for which we had already analyzed gene expression profiles for therapeutic applications; we found that they showed similar, but not the same, differentiation potential to form cardiomyocytes and other types of mature cells. Recently, we also noticed that, even between the different iPSC lines, heterogeneity had been raised as an issue in terms of differentiation efficiency $(6,7)$. To obtain a high differentiation yield into cardiomyocytes for a therapeutic application or an in vitro assay, we need a better understanding of biomarkers to predict cardiac differentiation from PSCs (8). Moreover, in respect to cardiac differentiation, if we have an insight into a key molecule of the cardiac biomarker, we can select a better cell line among various PSCs to get better efficiency in cardiomyocytes differentiation.

In the present study, to find a pivotal molecule to predict cardiac differentiation from the pluripotent state, we compared two different types of pluripotent stem cells, ESC versus iPSC, showing different efficiency in cardiac differentiation.

\section{RESULTS}

Similarity in pluripotency gene expression and difference in proliferation between iPSC and ESC

We previously reported the generation of iPSC from fibroblasts of mouse skin (FVB strain) or mouse hearts (C57BL/6 strain) following treatment of fibroblasts with protein lysate of $\mathrm{mESC}$ (9). We named this iPSC as protein-engineered iPSC (PE-iPSC). We compared PE-iPSC (passage 50-60) derived from skin fibroblasts of a FVB mouse (sFB-iPSC) and mESC (passage 50-60) from a C57BL/6 mouse (C57-mESC). In terms of morphology, both PSCs showed typical dome-shaped colonies, which indicated morphological pluripotency, on maintenance culture (Supplementary Fig. S1A). We also confirmed vivid expression of a stemness gene, Oct4, in the nucleus of both stem cells (Supplementary Fig. S1B). Both expressed not only Oct4, but also other pluripotency markers, 
including Nanog, Rex1 and Sox2 to similar levels by semiquantitative RT-PCR (Supplementary Fig. S1C). Quantitative RT (Real Time)-PCR result confirmed the high expression of Oct4 and Nanog in both PSCs (Supplementary Fig. S1D).

However, sFB-iPSC showed slightly faster proliferation rate than C57-mESC on maintenance culture (Supplementary Fig. $\mathrm{S} 1 \mathrm{E}$ and $\mathrm{F}$ ). Also, we confirmed the faster proliferation of sFBiPSC than that of C57-mESC using WST-1 assay (Supplementary Fig. S1G).

\section{Both stem cells can be spontaneously differentiated into CMC, SMC, and EC, but SFB-iPSC produced more CMC and beating foci than $\mathrm{C} 57-\mathrm{mESC}$}

Next, we compared the natural differentiation abilities between sFB-iPSC and C57-mESC using an embryoid body (EB)-based differentiation protocol (Fig. 1A). We made comparable-sized EB from both PSCs and then attached them to $0.1 \%$ gelatin-coated dish at day 7 . We cultured them until day 17 when foci of spontaneous beating emerged (Fig. 1B). At day 17, we confirmed differentiation from the PSCs into cardiomyocytes (CMC), smooth muscle cells (SMC) and endothelial cells (EC) by immunofluorescent staining (Fig. 1C).
As differentiation progressed, pluripotency marker (Oct4 and Nanog) expression was decreased (Fig. 1D). At the same time, cardiac marker genes [cardiac troponin $T$ (cTnT) and alpha-myosin heavy chain $(\alpha-M H C)$ ] appeared at day 15 in sFB-iPSC and C57-mESC (Fig. 1D). Until day 15, vascular genes, including alpha-smooth muscle actin $(\alpha S M A), S M 22$ alpha (SM22 $\alpha$ ), Pecam-1 and Flk-1, showed a biphasic change in both PSCs (Fig. 1D). To identify the exact number of each type of the cardiovascular lineages, we harvested the differentiated cells at day 17 and analyzed them with flow cytometry. sFB-iPSC showed a significantly higher fraction of cTnT-positive cells than C57-mESC in contrast to a similar portion of aSMA-positive or PECAM-1-positive cells (Fig. 1E). In line with the flow cytometry, sFB-iPSC produced the significantly higher number of beating foci than C57-mESC by EB-based spontaneous differentiation (Fig. 1F). We concluded that both could be spontaneously differentiated into the cardiovascular lineage, but sFB-iPSC had a better efficiency of cardiac lineage differentiation than C57-mESC.
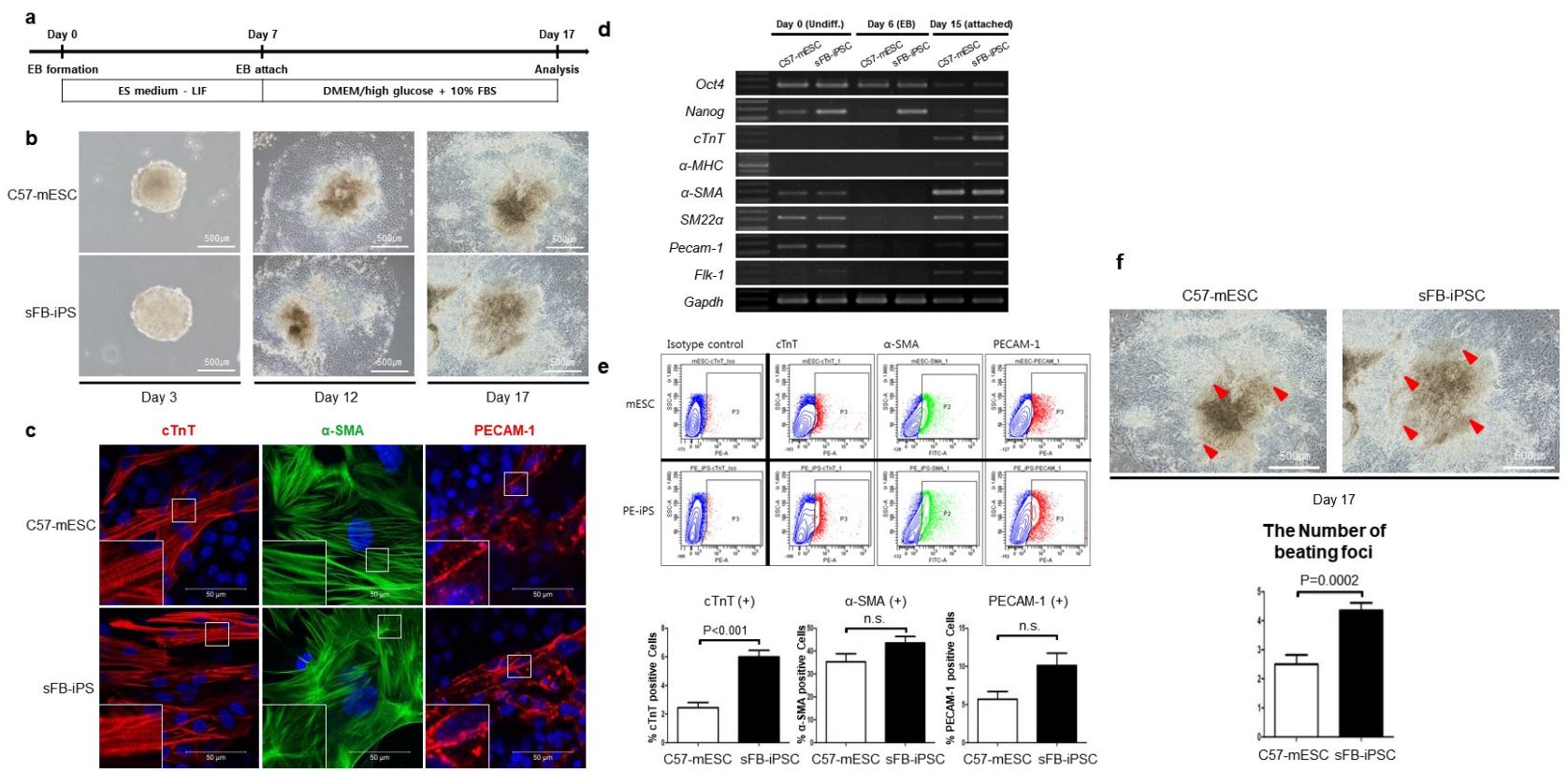

Fig. 1. Commitment to cardiac lineage during spontaneous differentiation of $m E B$ was higher in sFB-iPSC than C57-mESC. (A) Schematic diagram of embryoid body (EB)-based differentiation protocol. (B) Representative images of EB at day 3, attached EB at day 12, and emergence of beating foci at day 17. (C) Cardiomyocytes, smooth muscle cells and endothelial cells which differentiated from both pluripotent stem cells; CTnT: cardiac troponin T, $\alpha$-SMA: alpha smooth muscle actin, PECAM-1: platelet endothelial cell adhesion molecule-1. (D) Gene expression of C57-mESC and sFB-iPSC at an undifferentiated state (day 0), EB (day 6) and attached EB (day 15; 8 days after EB attachment). sFB-iPSC showed higher expression of $c T n T$. $\alpha$-MHC: alpha-myosin heavy chain ( $\mathrm{n}=3$ independent experiments, respectively). (E) Representative flow cytometry data comparing cTnT $(+)$, $\alpha-S M A(+)$, and PECAM-1 $(+)$ fraction between EB-based spontaneous differentiated C57-mESC and sFB-iPSC (upper panel) and its quantification (lower panel). cTnT( + ) cells were more in sFB-iPSC than in C57-mESC ( $n=8$ independent experiments). (F) Representative image of the attached EB from C57-mESC or sFB-iPSC with the emergence of beating foci at day 17 (left) and its quantification data (right). Red arrowheads denote beating foci that were more frequent in SFB-iPSC than in C57-mESC ( $\mathrm{n}=22$ technical replicates, respectively). Data are represented as mean \pm SD. 

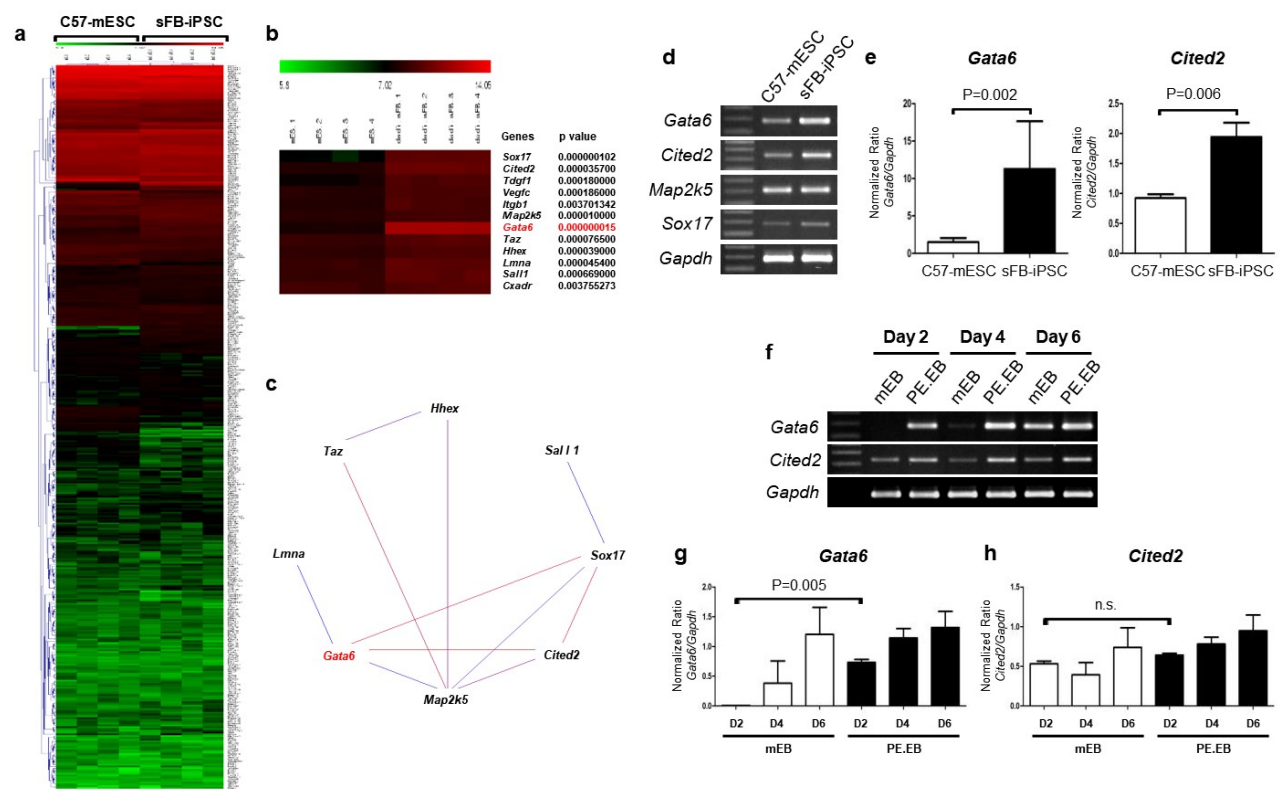

Fig. 2. Gene chip analysis of PSC at the undifferentiated status revealed that Gata6 was higher in sFB-iPSC than in C57-mESC. (A) The different expression level of genes that are related to heart development between C57-mESC and sFB-iPSC at the undifferentiated status by hierarchical clustering of microarray data. For the high-resolution figure, see Supplementary Fig. S2. (B) Top 12 genes whose expressions were higher in sFB-iPSC than in C57-mESC during spontaneous differentiation of EB. These genes are sorted by at least 2-fold differences of average expression between C57-mESC and sFB-iPSC. (C) The network diagram of 8 up-regulated genes which have at least one interaction among 12 genes listed above. (D) Gene expression of 4 candidate genes that have at least three interactions among 8 up-regulated genes from Fig. 2C in semi-quantitative RT-PCR. (E) Two genes of Gata6 and Cited2, out of 4 genes from Fig. 3D, whose expressions were higher in sFB-iPSC than in C57-mESC. It is confirmed by quantitative RT (Real Time)-PCR ( $\mathrm{n}=6$ independent experiments). (F-H) The expression level of Gata6 and Cited2 during spontaneous differentiation of EB [C57-mESC-derived EB (mEB) and sFB-iPSC-derived EB (PE.EB)], measured by (F) time-sequential semi-quantitative RT-PCR and $(G, H)$ in time sequential quantitative RT(Real Time)-PCR $(n=2$ independent experiments). Data are represented as mean \pm SD.

\section{sFB-iPSC expressed a higher level of Gata6 than C57-mESC from an undifferentiated state to EB stage}

We compared global gene expression profiles between two PSCs in the undifferentiated state. In general, global gene expression profiles were similar to each other, as shown in our previous report (9). But there were 186 genes differentially expressed between the two PSCs (50 genes up-regulated in C57-mESC, 136 genes up-regulated in sFB-iPSC) by selecting genes with over 2 folds changes in the microarray analysis (Fig. 2A and Supplementary Fig. S2). We chose a list of genes which are related to heart development from a gene ontology website (www.geneontology.org) and then identified 12 genes which were up-regulated more in sFB-iPSC than in C57-mESC in the undifferentiated state (Fig. 2B).

Among those 12 genes, Gata6 revealed the lowest p-value, which means the most statistically significant difference in expression level between two PSCs (Fig. 2B). Furthermore, by clustering those genes, we narrowed the candidate target genes down to 4 genes; Map2k5, Cited2, Gata6, and Sox17. Each one of these genes had an interaction with multiple genes (Fig. 2C). Among those 4 target genes having numerous interactions, quantitative RT-PCR data confirmed that Gata6 and Cited 2 were significantly up-regulated in sFB-iPSC than
C57-mESC, in contrast to Map2k5 and Sox17 (Fig. 2D and E). Gata6, in particular, was induced to a larger extent in sFB-iPSC compared to C57-mESC at the early stage during spontaneous differentiation of EB, while Cited2 was marginally increased in sFB-iPSC (Fig. 2F-H).

\section{Gata6 knock-down in iPSC decreased the efficiency of CMC production, but not that of SMC and EC}

We established stable clones of sFB-iPSC which were transduced with GFP-lentivirus expressing either non-targeted shRNA (shNT) or shRNA targeting Gata6 (shGata6) (Supplementary Fig. S3A and B). We confirmed that Gata6 knock-down efficiently suppressed Gata6 expression during EB formation (Fig. $3 \mathrm{~A}$ and B). The shNT and shGata6 transduction did not affect the expression of pluripotent marker genes in the clones as well (Fig. 3C). There was no difference in EB formation or growth after EB reattachment between the original sFB-iPSC and the shRNA transduced clones (Fig. 3D-F).

However, during spontaneous differentiation of $E B$ after reattachment, the non-transduced EB and shNT EB showed earlier expressions of $C T n T$ whereas shGata6 EB showed weak expression of $C T n T$ (Fig. 3G and H). The shGata6 clone showed a remarkable reduction in the proportion of cTnT-positive 


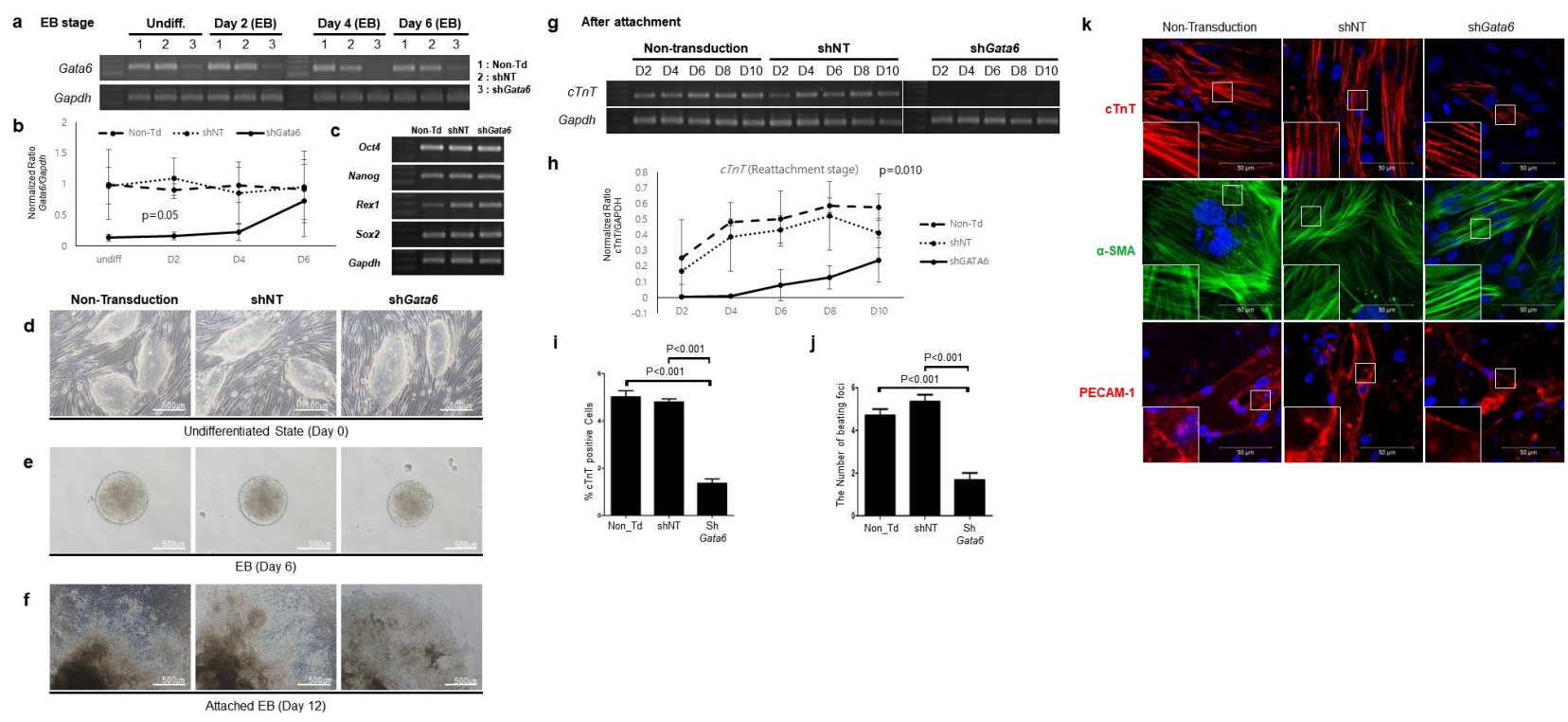

Fig. 3. Gata6 knock-down blunted cardiac differentiation of sFB-iPSC. (A, B) Time-sequential expression of Gata6 during spontaneous differentiation of EB before attachment in sFB-iPSC (Non-transduction, shNT, and shGata6), using (A) semi-quantitative RT-PCR, (B) quantitative RT(real Time)-PCR. Transduction of shGata6 effectively blocked the expression of Gata6 in sFB-iPSC during spontaneous differentiation. (C) Pluripotent marker genes expression in sFB-iPSC with shGata6 and its control groups at the undifferentiated status. (D-F) Representative morphologies of sFB-iPSC with Gata6 knocked-down using shGata6, comparing with control groups (Non-transduction and shNT) at (D) undifferentiated state Day 0, (E) EB Day 6 and (F) attached EB Day 12. $(\mathrm{G}, \mathrm{H})$ Time-sequential expression of $\mathrm{CTnT}$ as a cardiac marker in sFB-iPSC during spontaneous differentiation of EB after attachment (Non-transduction, shNT, and shGata6), using (G) semi-quantitative RT-PCR and (H) quantitative RT(Real Time)-PCR. ( $(n=3$ independent experiments for all data in Figure 4, respectively). Spontaneous differentiation to cardiomyocytes was blocked by shGata6 in sFB-iPSC. (I, J) Differentiation to cardiomyocytes was blunted in SFB-iPSC with Gata6 knocked-down. (I) The proportion of troponin T-positive cardiomyocytes measured by flow cytometry ( $\mathrm{n}=6$ independent experiments, respectively) and (J) the beating foci number after spontaneous differentiation of sFB-iPSC with shGata6 and its controls group $(n=43$ technical replicates, respectively). (K) Representative immunofluorescent images of the spontaneous differentiation of EB at the attached culture from sFB-iPSC with shGata6 and its control groups; cTnT, $\alpha$-SMA, PECAM-1 as a cardiomyocyte, smooth muscle cell, and endothelial cell marker, respectively. Data are represented as mean \pm SD.

cardiomyocytes (Fig. 3I, Supplementary Fig. S3A and B) and the number of beating foci, compared to the non-transduced sFB-iPSC or the shNT clone (Fig. 3J). In line with the flow cytometry analysis, the shGata6 clone showed less cTnTpositive cardiomyocytes and poor myofibril development compared to non-transduced sFB-iPSC or the shNT clone (Fig. 3K). Additionally, endothelial cell, and smooth muscle cell proportion are also decreased, but to a lesser extent than that of cardiomyocytes (Supplementary Fig. S3A and B).

Variable expression levels of Gata6 among stable PSC lines, correlated with the potential to differentiate into cardiomyocytes

We established several clones of C57-mESC that originated from single cell clones. We found variations in Gata6 expression levels among the single cell clones of C57-mESC. Among the 6 clones, the number 5 clone had a significantly higher level of Gata6 than the other 5 clones (Fig. 4A). We compared the cardiac differentiation ability of the clone no. 5 with the highest Gata6 level and the clone no.3 with the lowest. The clone no. 5 resulted in a higher number of beating foci than the clone no.3 (Fig. 4B).
To test whether a higher level of Gata6 is a typical feature of iPSC, other than $\mathrm{mESC}$, or whether it is a universal indicator of a better potential for cardiac lineage, we analyzed additional $\mathrm{mESC}$ and iPSC lines. Interestingly, mESC from E14 mouse (E14-mESC) expressed Gata6 gene three times higher than sFB-iPSC, as well as PE-iPSC derived from cardiac fibroblast of the C57BL/6 mouse (cFB-iPSC) (Fig. 4C). Then, we compared E14-mESC and CFB-iPSC. As we expected, regardless of whether it is $\mathrm{mESC}$ or PE-iPSC, the higher level of the Gata6 level was essential to make the number of beating foci (Fig. 4D and $\mathrm{E})$.

\section{DISCUSSION}

sFB-iPSC differentiated into cardiomyocytes more efficiently than C57-mESC. After we compared global gene expression profiles, we found that Gata6 was sustainably up-regulated in sFB-iPSC. When Gata6 was down-regulated in sFB-iPSC, the efficiency of cardiac differentiation was markedly reduced. We confirmed the importance of Gata6, regardless of whether it is $\mathrm{mESC}$ or iPSC, by validation experiments using single cell clones with different Gata6 expression and other PSC lines 
a

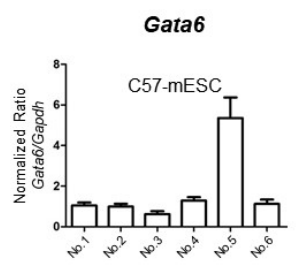

c
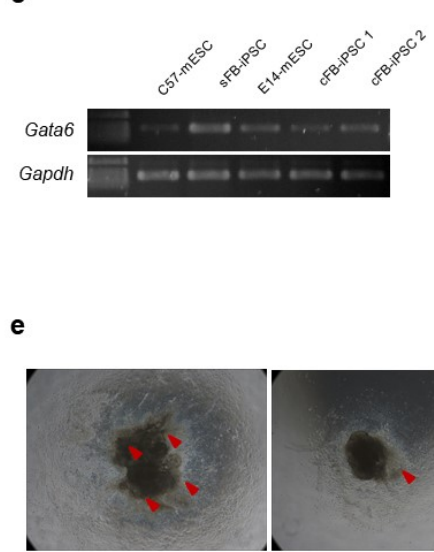

E14-mESC b

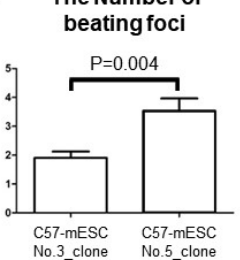

d
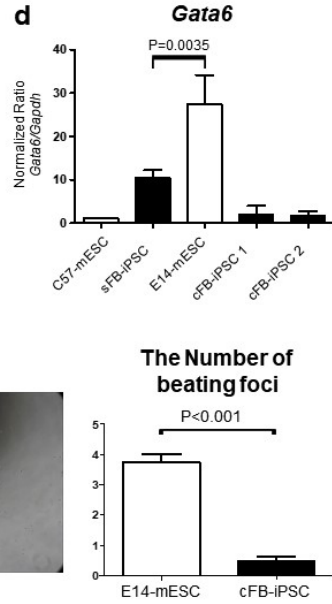

Fig. 4. Consistent correlation between Gata6 level and the ability to differentiate toward cardiomyocytes among several different PSC lines. (A) Variable Gata6 expression levels among several stable cell lines established from a single colony of C57-mESC, using quantitative RT (Real Time)-PCR ( $\mathrm{n}=3$ independent experiments, respectively). (B) The different efficiency of cardiomyocyte differentiation by comparing the number of beating foci during spontaneous differentiation of C57-mESC stable cell lines between no.3 and no. 5, which showed the most significant difference of Gata6 expression level at undifferentiated state from Fig. 4A ( $\mathrm{n}=3$ independent experiments, respectively). (C, D) Gata6 expression level in various PSC lines including the other $\mathrm{mESC}$ and PE-iPSC lines by using (C) semi-quantitative RT-PCR and (D) quantitative RT(Real Time)-PCR ( $n=3$ independent experiments, respectively). (E) Representative image of beating foci at day 17 from the attached mEB (E14) with the highest Gata6 and PE.EB (Cardiac Fibroblast.1, cFB.1) with the lowest Gata6 (left) and its quantification data (right). Red arrowheads denote beating foci $(n=22$ technical replicates, respectively). Data are represented as mean $\pm \mathrm{SD}$.

originated from the other sources. These data suggest that the level of Gata6 expression could be a genetic marker in the undifferentiated state to select the pluripotent stem cells that have a better efficiency of cardiac differentiation.

For translational research or clinical application, pluripotent stem cell lines should reliably, efficiently, and stably differentiate into cardiomyocytes. However, the variation has been observed in the differentiation efficiency of various human ES cell lines: a neuronal differentiation variation in 6 human ESC lines (10), a marked propensity to differentiate into specific lineages of 17 human ESC lines (11). Furthermore, the equivalence of human ESC and iPSC lines have been questioned. For example, it has been reported that human iPSC differs from ESC in various points of view: the expression of hundreds of genes (12), the single-cell gene expression profiles of 362 human iPSC and ESC (13), their genome-wide DNA methylation patterns (14), and their neural differentiation properties (15). In a study (13), human iPSC displayed slower growth kinetics and impaired directed differentiation to endothelial cells or cardiomyocytes compared with human ESC. Those variations, or so-called heterogeneity of pluripotent stem cells, may be affected by the methods used for reprogramming or differentiation as well as the origin of the reprogrammed cells. Heterogeneity is also found in the different batches of the cell lines established by the same method in the same laboratory.

As a marker of the differentiation potential into a cardiomyocyte, we discovered Gata6 in pluripotent stem cells. Gata6 belongs to a family of zinc finger transcription factors that are transcriptional proteins regulating embryonic morphogenesis and tissue-specific differentiation. It has been known well as an essential gene in cardiac development. Morrisey and colleagues generated mice deficient in Gata6 by targeted disruption (16). Differentiation of embryoid bodies derived from Gata6 ${ }^{-1-}$ ESC lacked a covering layer of visceral endoderm and failed to express the genes encoding early and late endodermal markers, including $\mathrm{Hnf4}$ and Gata4. Homozygous Gata6 ${ }^{-1-}$ mice died between embryonic day 6.5 and 7.5 with a specific defect in endoderm differentiation. When Gata6 is inactivated only in vascular smooth muscle cells or in neural crest, it resulted in perinatal mortality from a spectrum of cardiovascular defects (17). Furthermore, heterozygous mice for either a Gata4 or Gata6 null allele are normal, but the double heterozygotes of Gata4 and Gata6 null alleles resulted in embryonic lethality by day 13.5 and was accompanied by a spectrum of cardiovascular defects (18). Double homo-knockout of both Gata4 and Gata6 led to the complete absence of heart (19), and GATA4, GATA5, and GATA6 could each efficiently enhance cardiogenesis in ESC derivatives, with some distinctions (20), suggesting that these GATA factors act cooperatively in cardiovascular development. The main finding of this study is that Gata6 expression in an undifferentiated state of PSCs affects the differentiation potential into cardiomyocytes. Therefore, we could use the Gata6 level as a selection marker for a PSC with high differentiation potential into cardiomyocytes among others. Many experimental trials have been conducted to increase therapeutic efficacy. These studies included separation of cardiac progenitors or the forced expression of cardiac transcription factors, which have their own limitations. Therefore, selecting an efficient stem cell line from various lines by a marker gene seems to be a fascinating way to improve the total efficiency of stem cell therapy. Gata6 would be an appropriate marker.

Even though various PSC lines showed a different level of Gata6 expression, it did not affect pluripotency maintenance. 
sFB-iPSC was also fully reprogrammed cells showing expression of pluripotency genes, teratoma formation and genetic profiles close to ESC compared to C57-mESC. In addition, cFB-iPSC with low expression of Gata6 did not show any abnormality in growth rate, morphology, or expression of pluripotency genes.

One of the limitations of our study is that we did not assess contractile function or electrical activity of the differentiated cardiomyocytes. Also, we evaluated the efficiency at a single time point, but long-term culture may show late catch-up growth.

In conclusion, cardiac differentiation efficiency varies depending on the PSC lines. Gata6 level at undifferentiated state is a marker predicting the capability of cardiac differentiation among different PSCs. Gata6 would help select a better pluripotent stem cell line for cardiac regenerative medicine.

\section{MATERIALS AND METHODS}

\section{Cell culture}

Induction of cardiac differentiation: We used embryoid body(EB)-based spontaneous differentiation protocol for cardiovascular differentiation (21). Briefly, undifferentiated $\mathrm{mESC}$ or PE-iPSC was detached by one minute incubation with $0.25 \%$ Trypsin/EDTA (Gibco). Then, we seeded the whole detached cells in a tissue culture plate and incubated $5 \% \mathrm{CO}_{2}$ atmosphere at $37^{\circ} \mathrm{C}$ for 30 mins. Thereafter, we gently collected floating cells in the supernatant, which is considered as $\mathrm{mESC} / \mathrm{PE}-\mathrm{iPSC}$ in passaging. Next, we seeded them at $2 \times$ $10^{4} / \mathrm{cm}^{2}$ in the ES medium without LIF on a petri dish. After one day, EBs were formed. We changed the medium every other day. Seven days after EB formation, we reattached the EBs on $0.1 \%$ gelatin-coated plate. Then, cells grew out radially from the EBs. The sprouting cells differentiated into a heterogeneous population of cells including beating cardiomyocytes. Quantification of beating foci: To quantify the number of beating foci, we seeded one EB for each well of a 96 well plate. After 5 days from reattachment, we counted the number of beating foci of each well and compared the mean number of beating foci per well between cell lines.

\section{Comparison of proliferation between ESC and iPSC}

We evaluated proliferation of each pluripotent stem cells using the WST-1 kit (Roche) as well as estimating a cumulative number of cells. For the WST-1 assay, we seeded $1 \times 10^{3}$ undifferentiated ESC/PE-iPSC on mitotically inactivated STO cells on a 96-well plate. We performed a WST-1 assay on 3 days after seeding and analyzed each sample using 450nm absorbance by Gemini EM fluorescence microplate reader (Molecular Devices). To calculate the cumulative number of cells, $2 \times 10^{5}$ cells were seeded on prepared STO feeder layer. After 5 days, cells were detached with $0.25 \%$ Trypsin/EDTA (Gibco) and pre-plated for 30 minutes on a tissue culture plate to remove feeder cells. Then, we collected the supernatant gently and counted the number of cells. We repeated this step every passage. Then, we calculated the cumulative number of cells as the following formula: $\mathrm{CN}_{\mathrm{n}}=\left(\mathrm{CN}_{\mathrm{n}-1} * \mathrm{~N}_{\mathrm{n}}\right) / 2 * 10^{5}\left(\mathrm{CN}_{\mathrm{n}}\right.$; cumulative cell number at passage $n, N_{n}$; the counted number of cells at passage $n$ )

\section{Microarray}

Global gene expression analyses were performed using Affymetrix Gene-Chip Mouse Gene 1.0 ST oligonucleotide arrays (Affymetrix) or Mouse Whole-genome BeadChips (Illumina). The sample preparation was performed according to the instructions provided by the manufacturer (22). Microarray results are accessible at the Gene Expression Omnibus (GEO) database (National Center for Biotechnology Information; accession no. series GSE13770). We used Multiple Experiment Viewer program (MeV, The TM4 development group), a Java tool for genomic data analysis to get an intuitive graphical interface for clustering, classification, statistical analysis. We discovered several genes related to heart development to generate a hypothesis which could explain why sFB-iPSC showed better differentiation efficiency into cardiomyocytes than c57-mESC.

\section{shRNA knock-down experiment}

We utilized the lentiviral vector pLentiLox 3.7 (pLL3.7, Addgene plasmid 11795) (23) expressing the mouse scrambled shRNA or shGata6 (sequence Hpal-shGata6-5p-F TG-CGGTC TCTACAGCAAGATG-TTCAAGAGA-CATCTTGCTGTAGAGA CCG-CTTTTTTC, Xhol-shGata6-5p-R TCGAGAAAAAAG-CGG TCTCTACAGCAAGATG-TCTCTTGAA-CATCTTGCTGTAGAG ACCG-CA) (Sigma Aldrich) which has GFP as a selection marker. For the generation of lentivirus, we transfected HEK293T cell with $20 \mu \mathrm{g}$ of a lentiviral vector, $10 \mu \mathrm{g}$ of PLP1 (Invitrogen), $10 \mu \mathrm{g}$ of PLP2 (Invitrogen), $10 \mu \mathrm{g}$ of VSVG (Invitrogen) using a transfection agent, polyethyleneimine (Polysciences). After 24 hours, we changed the medium with DMEM/high glucose (Gibco) supplemented with 10\% FBS (Gibco). After 24 hours, we harvested the supernatant and added $10 \mathrm{ml}$ of growth medium for the second harvest. For lentivirus transduction, we prepared retronectin (TaKaRa, Japan)-coated $35 \mathrm{~mm}$ dish. We seeded $2 \times 10^{5}$ PE-iPSC or $\mathrm{mESC}$ on the dish with $1.5 \mathrm{ml}$ virus supernatant and $1.5 \mathrm{ml}$ fresh growth medium. After 24 hours, we collected the whole cells in the dish and re-seeded on a dish with STO feeder layer. After 7 days, we mechanically selected undifferentiated colonies expressing GFP. We dissociated the colonies with $0.25 \%$ Trypsin/EDTA (Gibco) and then seeded them on a dish with STO feeder layer.

\section{Statistical analysis}

All results are expressed as the mean \pm standard deviation (SD). The differences of continuous variables between experimental groups were analyzed by Student's t-test or ANOVA test using GraphPad Prism 5 (GraphPad software). 


\section{Ethical statement}

Seoul national university hospital Institutional Animal Care and Use Committee (IACUC) approved this research (approval number: 10-0046).

\section{ACKNOWLEDGEMENTS}

HS Kim was supported by the Korea Health Technology R\&D Project (HI14C1277) through the Korea Health Industry Development Institute (KHIDI), funded by the Ministry of Health \& Welfare (MHW). CH Yoon was supported by the Industrial Strategic technology development program (10052980) funded by the Ministry of Trade, Industry \& Energy of Korea and the Korean Health Technology R\&D Project.

\section{CONFLICTS OF INTEREST}

The authors have no conflicting interests.

\section{REFERENCES}

1. Kim PH, Cho JY (2016) Myocardial tissue engineering using electrospun nanofiber composites. BMB Rep 49, 26-36

2. Tongers J, Losordo DW and Landmesser U (2011) Stem and progenitor cell-based therapy in ischaemic heart disease: promise, uncertainties, and challenges. Eur Heart J 32, 1197-1206

3. Zhang J, Wilson GF, Soerens AG et al (2009) Functional cardiomyocytes derived from human induced pluripotent stem cells. Circ Res 104, e30-41

4. Narazaki G, Uosaki H, Teranishi M et al (2008) Directed and systematic differentiation of cardiovascular cells from mouse induced pluripotent stem cells. Circulation 118, 498-506

5. Nelson TJ, Martinez-Fernandez A, Yamada S, Perez-Terzic C, Ikeda Y and Terzic A (2009) Repair of acute myocardial infarction by human stemness factors induced pluripotent stem cells. Circulation 120, 408-416

6. Kaichi S, Hasegawa K, Takaya T et al (2010) Cell line-dependent differentiation of induced pluripotent stem cells into cardiomyocytes in mice. Cardiovasc Res 88, 314-323

7. Streckfuss-Bomeke K, Wolf F, Azizian A et al (2013) Comparative study of human-induced pluripotent stem cells derived from bone marrow cells, hair keratinocytes, and skin fibroblasts. Eur Heart J 34, 2618-2629

8. Burridge PW, Keller G, Gold JD and Wu JC (2012) Production of de novo cardiomyocytes: human pluripotent stem cell differentiation and direct reprogramming. Cell Stem Cell 10, 16-28

9. Cho HJ, Lee CS, Kwon YW et al (2010) Induction of pluripotent stem cells from adult somatic cells by protein-based reprogramming without genetic manipulation. Blood 116, 386-395
10. Di Giorgio FP, Boulting GL, Bobrowicz S and Eggan KC (2008) Human embryonic stem cell-derived motor neurons are sensitive to the toxic effect of glial cells carrying an ALS-causing mutation. Cell Stem Cell 3, 637-648

11. Osafune K, Caron L, Borowiak M et al (2008) Marked differences in differentiation propensity among human embryonic stem cell lines. Nat Biotechnol 26, 313-315

12. Chin MH, Mason MJ, Xie W et al (2009) Induced pluripotent stem cells and embryonic stem cells are distinguished by gene expression signatures. Cell Stem Cell 5, 111-123

13. Narsinh KH, Sun N, Sanchez-Freire V et al (2011) Single cell transcriptional profiling reveals heterogeneity of human induced pluripotent stem cells. J Clin Invest 121, 1217-1221

14. Doi A, Park IH, Wen B et al (2009) Differential methylation of tissue- and cancer-specific $\mathrm{CpG}$ island shores distinguishes human induced pluripotent stem cells, embryonic stem cells, and fibroblasts. Nat Genet 41 , 1350-1353

15. Hu BY, Weick JP, Yu J et al (2010) Neural differentiation of human induced pluripotent stem cells follows developmental principles but with variable potency. Proc Natl Acad Sci U S A 107, 4335-4340

16. Morrisey EE, Tang Z, Sigrist K et al (1998) GATA6 regulates HNF4 and is required for differentiation of visceral endoderm in the mouse embryo. Genes Dev 12, 3579-3590

17. Lepore JJ, Mericko PA, Cheng L, Lu MM, Morrisey EE and Parmacek MS (2006) GATA-6 regulates semaphorin 3C and is required in cardiac neural crest for cardiovascular morphogenesis. J Clin Invest 116, 929-939

18. Xin M, Davis CA, Molkentin JD et al (2006) A threshold of GATA4 and GATA6 expression is required for cardiovascular development. Proc Natl Acad Sci U S A 103, 11189-11194

19. Zhao R, Watt AJ, Battle MA, Li J, Bondow BJ and Duncan SA (2008) Loss of both GATA4 and GATA6 blocks cardiac myocyte differentiation and results in acardia in mice. Dev Biol 317, 614-619

20. Turbendian HK, Gordillo M, Tsai SY et al (2013) GATA factors efficiently direct cardiac fate from embryonic stem cells. Development 140, 1639-1644

21. Maltsev VA, Wobus AM, Rohwedel J, Bader $M$ and Hescheler J (1994) Cardiomyocytes differentiated in vitro from embryonic stem cells developmentally express cardiac-specific genes and ionic currents. Circ Res 75, 233-244

22. Kim JO, Kwon EJ, Song DW, Lee JS, Kim DH (2016) miR-185 inhibits endoplasmic reticulum stress-induced apoptosis by targeting $\mathrm{Na}+/ \mathrm{H}+$ exchanger- 1 in the heart. BMB Rep 49, 208-213

23. Rubinson DA, Dillon CP, Kwiatkowski AV et al (2003) A lentivirus-based system to functionally silence genes in primary mammalian cells, stem cells and transgenic mice by RNA interference. Nat Genet 33, 401-406 